\title{
The Craig Interpolation Theorem in abstract model theory
}

\author{
Jouko Väänänen
}

Received: 12 March 2008 / Accepted: 2 June 2008 / Published online: 1 July 2008

(C) The Author(s) 2008

\begin{abstract}
The Craig Interpolation Theorem is intimately connected with the emergence of abstract logic and continues to be the driving force of the field. I will argue in this paper that the interpolation property is an important litmus test in abstract model theory for identifying "natural," robust extensions of first order logic. My argument is supported by the observation that logics which satisfy the interpolation property usually also satisfy a Lindström type maximality theorem. Admittedly, the range of such logics is small.
\end{abstract}

Keywords Interpolation - Abstract logic - Generalized quantifier . Infinitary language

\section{Introduction}

Abstract model theory studies general model theoretic properties of extensions of first order logic obtained by adding generalized quantifiers, infinitary operations, or higher order quantifiers. We will not define the concept of an abstract logic here, as the definition is quite long and the intuitive concept suffices for this survey. The intuitive concept is the following: In first order logic every sentence $\varphi$ has an associated class $K_{\varphi}$ of models, namely the class of models of $\varphi$. If we forget about the sentences $\varphi$ we can think of first order logic simply as a collection $\mathcal{L}$ of model classes $K$. First order logic has thus become an abstract logic. More generally an abstract logic is a collection $\mathcal{L}$ of model classes $K$ satisfying some natural axioms such as every model class

\footnotetext{
J. Väänänen $(\varangle)$

ILLC, University of Amsterdam, Plantage Muidergracht 24, 1018 TV Amsterdam, The Netherlands e-mail: vaananen@science.uva.nl; jouko.vaananen@helsinki.fi

J. Väänänen

Department of Mathematics and Statistics, University of Helsinki, Helsinki, Finland
} 
$K$ is closed under isomorphisms, the collection is closed under intersections $K \cap K^{\prime}$ and complements $-K$, etc. This is similar to defining what it means for a subset of the Euclidean space $\mathbb{R}^{3}$ to be open, and then abstracting from this the concept of a topology: any collection of sets, closed under finite intersections and arbitrary unions. For a thorough definition of the concept of abstract logic, see Barwise and Feferman (1985).

We use $\mathcal{L}, \mathcal{L}^{\prime}$, etc. to denote abstract logics, and $\varphi, \psi, \theta$, etc. to denote sentences of abstract logics. Vocabularies are denoted by $L, L^{\prime}$, etc. The vocabulary of a sentence $\varphi$ is denoted $\tau(\varphi)$. Suppose $\mathcal{L}$ is a logic and $L$ a vocabulary. A class $K$ of $L$-structures is an $\operatorname{EC}(\mathcal{L})$-class if there is a sentence $\varphi \in \mathcal{L}$ with $\tau(\varphi)=L$ such that $K$ is the class of all models of $\varphi$. We then say alternatively that $K$ is an $\mathcal{L}$-definable class. A class $K$ of $L$-structures is a $\operatorname{PC}(\mathcal{L})$-class if there is a vocabulary $L^{\prime} \supseteq L$ and a class $K^{\prime}$ of $\mathcal{L}^{\prime}$-structures such that $K^{\prime}$ is $\mathcal{L}$-definable and $K=\left\{\mathfrak{M} \uparrow L: \mathfrak{M} \in K^{\prime}\right\}$. A class $K$ of $L$-structures is an $\operatorname{RPC}(\mathcal{L})$-class if there is a vocabulary $L^{\prime} \supseteq L$, a unary predicate $P \in L^{\prime}$ and a class $K^{\prime}$ of $\mathcal{L}^{\prime}$-structures such that $K^{\prime}$ is $\mathcal{L}$-definable and $K=\left\{\mathfrak{M}^{\left(P^{\mathfrak{M}}\right)} \uparrow L: \mathfrak{M} \in K^{\prime}\right\}$

What kind of abstract logics, i.e. model theoretically defined extensions of first order logic, are there? We list in the Appendix concrete examples of abstract logics that are discussed in this paper. By and large the known extensions fall into three categories, see Fig. 1.

The first category is the class of higher order logics, most notably the second order $\operatorname{logic} L^{2}$, which permits quantification over arbitrary relations of the universe. The second category of extensions of first order logic are the countably compact extensions of $L_{\omega \omega}$ by generalized quantifiers, such as

$$
\begin{aligned}
& Q_{1} x \varphi(x) \Longleftrightarrow|\{a: \varphi(a)\}| \geq \aleph_{1} \\
& \left.Q_{\omega}^{c o f} x y \varphi(x, y) \Longleftrightarrow \operatorname{cof}(\{(a, b): \varphi(a, b))\}\right)=\omega \\
& Q_{1}^{M M} x y \varphi(x, y) \Longleftrightarrow \exists X\left(|X| \geq \aleph_{1} \text { and } \forall x, y \in X \varphi(x, y)\right)
\end{aligned}
$$

The countable compactness of $L_{\omega \omega}\left(Q_{1}^{M M}\right)$ is independent of ZFC, so this logic is not in the same category as $L_{\omega \omega}\left(Q_{1}\right)$ and $L_{\omega \omega}\left(Q_{\omega}^{c o f}\right)$, although it is, if we assume $\diamond$.

Fig. 1 Abstract model theory

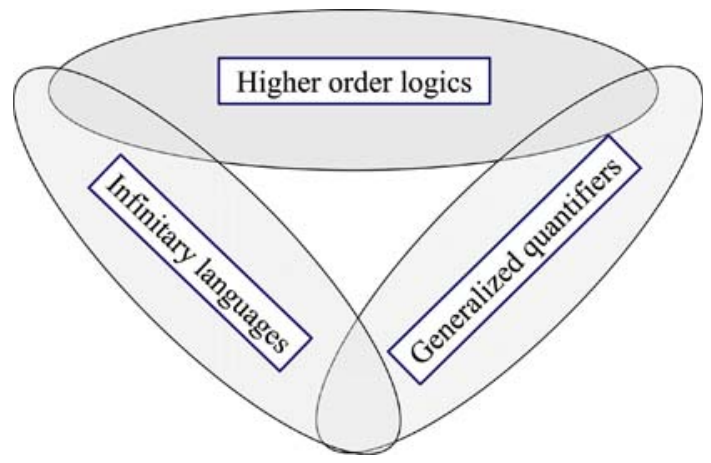


The third category of logics is the class of infinitary logics. Let $L_{\kappa \lambda}$ denote the logic in which conjunctions and disjunctions of length $<\kappa$ are allowed, as well as homogeneous strings of quantifiers of length $<\lambda$. The most studied of these logics is $L_{\omega_{1} \omega}$ and its admissible fragments $L_{A}$.

There is a special family of abstract logics which do not have any apparent syntax, just the semantics. These are the so called $\Delta$-extensions of abstract logics. The $\Delta$-extension of $\mathcal{L}$ is the smallest extension of $\mathcal{L}$ to a logic $\mathcal{L}^{\prime}$ such that $\operatorname{SK}\left(\mathcal{L}^{\prime}\right)$ (see below) holds. Alternatively, $\Delta(\mathcal{L})$ can be defined as the abstract logic, the sentences of which are exactly pairs $\left(K, K^{\prime}\right)$ of $\operatorname{RPC}(\mathcal{L})$-classes that are complements of each other. The nice thing about $\Delta(\mathcal{L})$ is that it always satisfies the Souslin-Kleene interpolation property, it has the countable compactness property if $\mathcal{L}$ does, and it has the Löwenheim property if $\mathcal{L}$ has. The downside is that in many important cases we do not have a natural syntax for $\Delta(\mathcal{L})$. This is the case, for example, with $\Delta\left(L\left(Q_{1}\right)\right)$.

Figure 2 displays the landscape of abstract logics. To the left ascends the "infinitary trail" and to the right the "quantifier trail." Both trails have positive occurrences of (relative) interpolation, but also "deathtraps." The trap in the infinitary trail is that at some point well-ordering becomes definable and everything is lost as far as interpolation is concerned (see below) in the sense that we have very strong counterexamples to interpolation and even to its weakest variants. The trap on the quantifier trail is that to find an extension with interpolation, of even the simplest logic $L\left(Q_{1}\right)$, one has to go beyond logics that are absolute with respect to CCC extensions of the universe. Thus such logics cannot be based on any simple property of $\aleph_{1}$ or $\aleph_{2}$. Above these trails hovers the realm of higher order logics, where model theory becomes set theory.

I have put the extension $L_{\omega \omega}\left(Q_{0}\right)$ of $L_{\omega \omega}$ with the quantifier

$$
Q_{0} x \varphi(x) \Longleftrightarrow|\{a: \varphi(a)\}| \geq \aleph_{0}
$$

on the infinitary trail, because it actually is very close to the smallest admissible fragment $L_{A}$ in the sense that $\Delta\left(L\left(Q_{0}\right)\right)=L_{A}$. In Fig. 2, I call the infinitary logics

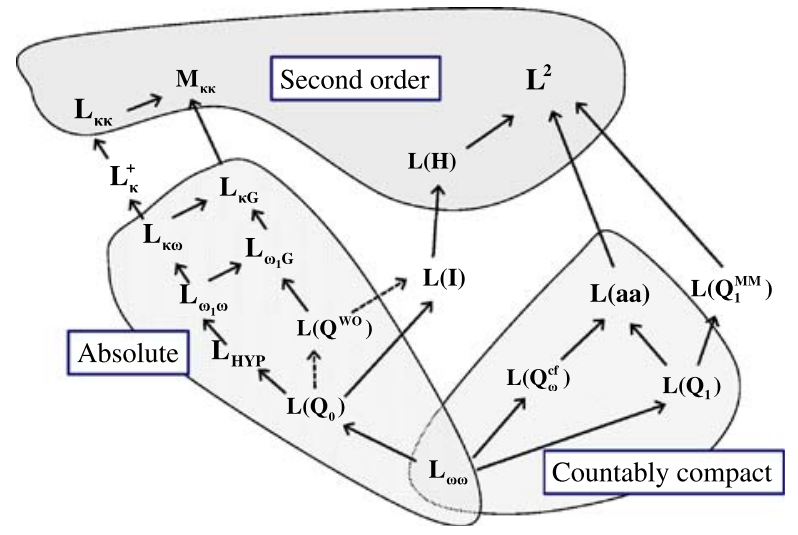

Fig. 2 The landscape 
up to $L_{\kappa \omega}$ and the extension $L_{\kappa G}$ of $L_{\kappa \omega}$ by the game quantifier

$$
\forall x_{0} \exists x_{1} \forall x_{2} \exists x_{3} \ldots \bigwedge_{n<\omega} \varphi_{n}\left(x_{0}, \ldots, x_{2 n+1}\right)
$$

absolute, following (Barwise 1972) as their syntax and semantics are absolute with respect to transitive models of set theory. By Theorem 1 first order logic is the only logic in the intersection of the infinitary trail and the quantifier trail.

The extension $L(H)$ of first order logic by the Henkin quantifier

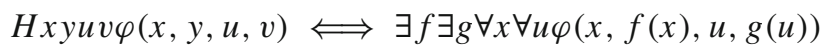

is essentially of the same strength as $L^{2}$ in the sense that $\Delta(L(H))=\Delta\left(L^{2}\right)$. The infinite quantifier logics $L_{\kappa \kappa}, \kappa>\omega$ are likewise essentially of second order nature, even if they allow quantification over subsets of size $<\kappa$ only.

The defining moment of abstract model theory was 1967 when Lindström (1969) proved:

Theorem 1 (Lindström's Theorem) First order logic $L_{\omega \omega}$ is maximal with respect to the Löwenheim property ${ }^{1}$ and the countable compactness property. ${ }^{2}$

There are many variations of this result and several closely related but different characterizations of first order logic. For a complete account of them, see Flum (1985). One of the main open questions of this field is whether the Löwenheim property can be replaced by the Craig Interpolation Property:

Interpolation property: If $\varphi$ and $\psi$ are sentences of $\mathcal{L}$ such that $\varphi \models \psi$, then there is an $\mathcal{L}$-sentence $\theta$ such that $\varphi \models \theta, \theta \models \psi$ and $\tau(\theta) \subseteq \tau(\varphi) \cap \tau(\psi)$.

This property is due to W. Craig (1957a). The relativized version $\operatorname{Craig}\left(\mathcal{L}, \mathcal{L}^{\prime}\right)$ stipulates that if such $\varphi$ and $\psi$ are taken from $\mathcal{L}$, then such a $\theta$ can be found in $\mathcal{L}^{\prime}$. Craig (1957b) showed that his interpolation theorem has an equivalent formulation as a separation property:

Separation property: Any two disjoint $\mathrm{PC}(\mathcal{L})$-classes can be separated by an $\mathrm{EC}(\mathcal{L})$-class.

Addison $(1962,2004)$ noted that the separation property is much like the separation property of the projective hierarchy of descriptive set theory and the Kleene hierarchies in recursive function theory, and suggested an approach to definability theory that would cover all three cases. Subsequently Vaught (1973) developed extensively the theory of $L_{\omega_{1} \omega}$ as an invariant version of descriptive set theory (see also Burgess 1977).

\footnotetext{
1 Every sentence with a model has a countable model.

2 Every countable set of sentences, every finite subset of which has a model, has itself a model. Logics with this property are called countably compact.
} 
While the separation property is clearly equivalent to the interpolation property, there are also strictly weaker versions. The Beth Definability Property, $\operatorname{Beth}(\mathcal{L})$, stipulates that if a sentence $\varphi \in \mathcal{L}$, with a predicate $P$ in $\tau(\varphi)$, has at most one interpretation for $P$ in every model of $\varphi$, then there is a formula $\psi \in \mathcal{L}$, with $\tau(\psi) \subseteq \tau(\varphi) \backslash\{P\}$, such that $\psi$ defines $P$ in every model of $\varphi$. As pointed out by Craig in his original paper, $\operatorname{Craig}(L)$ implies $\operatorname{Beth}(\mathcal{L})$. The Weak Beth Definability Property, WBeth $(\mathcal{L})$, is the variant of $\operatorname{Beth}(\mathcal{L})$ obtained by replacing "at most one" in the definition of the property by "exactly one." Naturally $\operatorname{Beth}(\mathcal{L})$ implies WBeth $(\mathcal{L})$. By $\operatorname{WBeth}\left(\mathcal{L}, \mathcal{L}^{\prime}\right)$ we mean the variant in which $\varphi \in \mathcal{L}$ and $\psi \in \mathcal{L}^{\prime}$. Finally, the Souslin-Kleene Interpolation Property, $\operatorname{SK}(\mathcal{L})$, is the special case of the separation property in which the two disjoint $\mathrm{PC}(\mathcal{L})$-classes are assumed to be complements of each other. Thus $\operatorname{Craig}(\mathcal{L})$ implies $\operatorname{SK}(\mathcal{L})$, and it is not hard to see that $\operatorname{SK}(\mathcal{L})$ implies $\operatorname{WBeth}(\mathcal{L})$. We have the "diamond of interpolation properties"-diagram of Fig. 3. None of the arrows can be reversed.

There are essentially three proofs of the Craig Interpolation Theorem known today: (1) Proof theoretic: based on Cut-Elimination and the Subformula Property. (2) Model theoretic: based on large homogeneous models. (3) Game theoretic/set theoretic: based on undefinability of well-order. It is arguable whether they are really fundamentally different proofs. In any case, attempts have been made to extend all of these proofs to extensions of first order logic. The model theoretic proof uses compactness in an essential way and is appropriate candidate in the quantifier trail where logics are in general countably compact. The game theoretic/set theoretic proof is appropriate in the infinitary trail up to the points $\left(L_{\omega_{1} \omega_{1}}\right.$ and $\left.L_{\omega_{1} G}\right)$ where well order becomes definable.

In the late 1960s both Mostowski (1968) and Lindström (1969) were contemplating the question why some extensions of first order logic satisfy the Craig Interpolation Theorem and others do not. They both came up with the general concept of what is now called abstract logic and showed that certain abstract logics do not satisfy interpolation. Mostowski gave a recursion theoretic criterion satisfied by weak second order logic and $L\left(Q_{0}\right)$ which is sufficient for an abstract logic to fail to satisfy interpolation.

Fig. 3 The diamond of interpolation properties

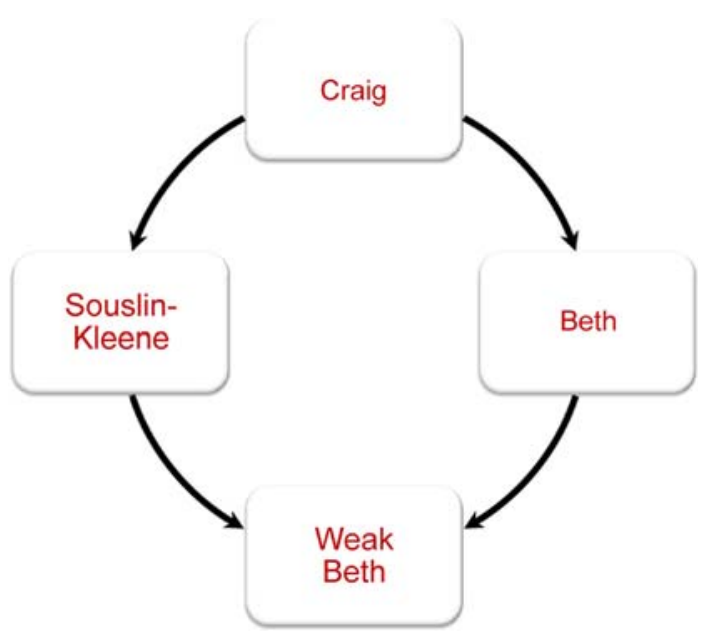


Lindström looked for a proof of the Beth Definability Theorem for some extensions of first order logic and came up with a new proof of the Robinson Consistency Property which eventually was transformed into his famous Theorem $1 .{ }^{3}$ These early developments emphasize the close relationship between the Craig Interpolation Theorem and the emergence of abstract model theory.

\section{The quantifier trail}

The quantifier trail of the landscape of Fig. 2 splits at first order logic into two parts: one to the logic $L\left(Q_{1}\right)$, based on the concept of countable cardinality, and one to the logic $L\left(Q_{\omega}^{\text {cof }}\right)$, based on the concept of countably cofinality. These two incomparable logics center around the two most basic concepts of set theory. The more elaborate concept of a stationary set has led to the logic $L(a a)$, where the branches from $L\left(Q_{1}\right)$ and $L\left(Q_{\omega}^{\text {cof }}\right)$ are again united.

It is an open problem whether there is any non-first order generalized quantifier $Q$ such that $L_{\omega \omega}(Q)$ satisfies the interpolation theorem. The problem is that the most likely candidates would be countably compact, but we do not know any examples of countable compact logics with interpolation, whether of the form $L_{\omega \omega}(Q)$ or not.

\subsection{Deathtraps}

Let us first discuss some problems among logics $L_{\omega \omega}(Q)$ which are not countably compact. The following fact was pointed out in Lindström (1969) and Mostowski (1968): Logics of the form $L_{\omega \omega}(Q)$ permit an implicit truth definition for themselves on $(\omega,<)$ ("adequate to truth in themselves" in the terminology of Feferman 1975). In some cases $L_{\omega \omega}(Q)$ can characterize $(\omega,<)$, for example if $Q$ is $Q_{0}, I$ or $H$. Then an undefinability of truth argument shows that $L_{\omega \omega}(Q)$-truth is implicitly but not explicitly definable on $(\omega,<)$. Thus in such a case $L_{\omega \omega}(Q)$ fails to satisfy the weak Beth property, and hence also the interpolation property. This simple but fundamental observation is not limited to logics of the form $L_{\omega \omega}(Q)$. Craig (1965) used this argument to refute interpolation in higher order logics. Gostanian and Hrbáček (1979) used this argument to refute interpolation in infinitary logic and to get strong failures of weak Beth for logics like $L_{\omega \omega}(I)$ and $L_{\omega \omega}(H)$, even $\neg \operatorname{WBeth}\left(L_{\omega \omega}(Q), L_{\infty \infty}(Q)\right)$ for $Q \in\{I, H\}$.

So if we want to find logics of the form $L_{\omega \omega}(Q)$ with interpolation, it makes sense to concentrate on those that are countably compact. For the logic $L\left(Q_{1}\right)$ a counterexample to interpolation was found by Keisler. It is the following. Let us consider a vocabulary with one binary relation symbol $E$. Let $K_{1}$ be the $\mathrm{PC}\left(L\left(Q_{1}\right)\right)$ class of equivalence relations with an uncountable set of non-equivalent elements, and $K_{2}$ the $\mathrm{PC}\left(L\left(Q_{1}\right)\right)$ class of equivalence relations with a countable set that meets every equivalence class. These model classes are disjoint but they cannot be separated in $L\left(Q_{1}\right)$, indeed not in any extension of $L_{\omega \omega}$ by unary generalized quantifiers. The proof

\footnotetext{
${ }^{3}$ For the story how Lindström came to the proof of his characterization of first order logic, see Lindström (1995).
} 
of this is an application of Ehrenfeucht-Fraïssé games for such logics (Caicedo 1983). Similar or more elaborate constructions show that interpolation fails in $L\left(Q_{\omega}^{\text {cof }}\right), L(a a)$ and $L\left(Q_{1}^{\mathrm{MM}}\right)$.

What about weaker form of interpolation for $L\left(Q_{1}\right)$, in the spirit of Fig. 3? Friedman (1973) showed, by utilizing Ehrenfeuch-Fraïssé games for $L\left(Q_{1}\right)$, that $L\left(Q_{1}\right)$ and many logics similar to it fail to satisfy the Beth property. Friedman asks what the situation is with the weak Beth property. For the answer, see below.

The following simple observation shows that nothing very simple will turn $L\left(Q_{1}\right)$ into a logic with interpolation:

Folklore fact: There is no abstract logic $\mathcal{L}$, absolute in $\mathrm{ZFC}$ between transitive models that preserve $\aleph_{1}$, that provably in ZFC satisfies $\operatorname{Sep}\left(L\left(Q_{1}\right), \mathcal{L}\right)$. More exactly, there are $\mathrm{PC}\left(L\left(Q_{1}\right)\right)$-classes $K_{1}$ and $K_{2}$, which are provably in ZFC disjoint, such that there is no term for an $\mathcal{L}$-sentence $\varphi$ such that truth of $\varphi$ is provably in ZFC preserved by $\mathrm{CCC}$ extensions, and $\varphi$ separates $K_{1}$ and $K_{2}$ provably in $\mathrm{ZFC}$.

Proof Let $K_{1}$ be the $\mathrm{PC}\left(L\left(Q_{1}\right)\right)$ class of tree-like (the set of predecessors of every element is linearly ordered) partial orders in which there is an uncountable branch, and $K_{2}$ the $\mathrm{PC}\left(L\left(Q_{1}\right)\right)$ class of tree-like partial orders in which there is mapping $f$ to a countable set such that the pre-image of every point is an antichain. These classes are $\mathrm{PC}\left(L\left(Q_{1}\right)\right)$-classes and disjoint, provably in ZFC. Since we assume $\operatorname{Sep}\left(L\left(Q_{1}\right), \mathcal{L}\right)$, there is a sentence $\varphi$ of $\mathcal{L}$ such that, provably in ZFC, $K_{1}$ is included in the class of models of $\varphi$, and no model of $\varphi$ is in $K_{2}$ (or $K_{1}$ and $K_{2}$ interchanged). Let us move to a forcing extension in which there is a Souslin tree $T$. Then $T$ is neither in $K_{1}$ nor in $K_{2}$. Case 1: $T$ satisfies, as a partial order, the sentence $\varphi$. Let us consider the generic extension $V[G]$ in which $T$ has been specialized with CCC forcing. In the extension, $T \in K_{2}$ and $T \models \varphi$, a contradiction. Case 2: $T$ satisfies as a partial order the sentence $\neg \varphi$. Let us consider the generic extension $V[G]$ in which a branch has been forced into $T$ with CCC forcing. In the extension, $T \in K_{1}$ and $T \models \varphi$, a contradiction.

\subsection{Positive results}

In the direction of generalized quantifiers the Gentzen approach has not been systematically studied and there may be some inherent reason for this. However, Alechina and van Lambalgen (1996) give a Gentzen system for $L\left(Q_{1}\right)$. Their system has cutelimination, but does not yield interpolation (of course, we know interpolation does not hold for $L\left(Q_{1}\right)$ ), because the cut-elimination does not give the needed subterm property. The stationary logic $L(a a)$ is another case where proof theory has been tried. After initial attempts to prove the Cut Elimination Theorem for the most obvious Gentzen system for $L(a a)$ failed, Szabo (1987) added new rules and proved the theorem. However, his system has an asymmetry which again prevents any conclusion in the direction of interpolation. This is a pity, for there is an interpolation property that $L(a a)$ has and that could perhaps have a Gentzen style proof, namely Shelah's (1985) relative interpolation theorem: 


$$
\operatorname{Craig}\left(L\left(Q_{\omega}^{\text {cof }}\right), L(a a)\right) \text {. }
$$

\subsubsection{The Robinson property}

In the model theoretic proof of interpolation for $L_{\omega \omega}$ one approach is to use the stronger version of interpolation, namely:

Robinson property: If $T$ and $T^{\prime}$ are two complete $\mathcal{L}$-theories such that all models of $T \cap T^{\prime}$ are $\mathcal{L}$-equivalent in the common vocabulary, then $T \cup T^{\prime}$ has a model.

The Robinson property can be proved for $L_{\omega \omega}$ by means of an alternating chain argument. However, there are even simpler proofs using large models. Suppose $\mathfrak{A}$ is a saturated ${ }^{4}$ model of $T$ and $\mathfrak{B}$ a saturated model of $T^{\prime}$ of the same cardinality as $\mathfrak{A}$. Let $L_{0}$ be the common vocabulary of $T$ and $T^{\prime}$. Then $\mathfrak{A} \uparrow L_{0}$ and $\mathfrak{B} \uparrow L_{0}$ are elementarily equivalent saturated models of the same cardinality, hence isomorphic. This isomorphism can be used to expand $\mathfrak{B}$ in a trivial way to a model of $T \cup T^{\prime}$. Essentially this proof, but with ultraproducts, was given by Keisler (1961).

The Robinson property implies the interpolation property for any logic: Suppose $\varphi \models \psi$. Let $T$ be a complete extension of the theory consisting of $\{\neg \psi\}$ and all logical consequences of $\varphi$ in the common vocabulary $L_{0}$. Let $T^{\prime}$ be a complete extension of the theory consisting of $\{\varphi\}$ and all elements of $T$ in the common vocabulary $L_{0}$. By the Robinson property, the theory $T \cup T^{\prime}$ is consistent, but this contradicts $\models \varphi \rightarrow \psi$. On the other hand, the interpolation property and compactness together give the Robinson property: Suppose $T \cup T^{\prime}$ has no models. Then by compactness, there is a finite conjunction $\varphi$ of sentences of $T$ and a finite conjunction $\psi$ of sentences of $T^{\prime}$ such that $\models \varphi \rightarrow \neg \psi$. Suppose $\theta$ is an interpolant. Let $\mathfrak{A} \models T$ and $\mathfrak{B} \models T^{\prime}$. Then $\mathfrak{A} \uparrow L_{0} \equiv \mathfrak{B} \uparrow L_{0}$, where $L_{0}$ is the common vocabulary. However, now $\mathfrak{A} \models \theta$, while $\mathfrak{B} \models \neg \theta$, a contradiction. In fact, the Robinson property also implies compactness (Makowsky and Shelah 1983), so Robinson property is equivalent to the combination of compactness and interpolation.

In the quantifier trail the most promising result about interpolation is Shelah's (1) above. It raises the intriguing question whether there is some logic $\mathcal{L}$ in between $L\left(Q_{\omega}^{\text {cof }}\right)$ and $L(a a)$ with interpolation. No such $\mathcal{L}$ is at the moment in sight. The proof of the fact (1) is a vast elaboration of the above proof of the Robinson property.

\subsubsection{The back-and-forth method}

The following game is due to Ehrenfeucht (1960): Let $L$ be a finite relational vocabulary and $\mathfrak{M}, \mathfrak{M}^{\prime} L$-structures such that $\mathfrak{M} \cap \mathfrak{M}^{\prime}=\emptyset$. We use $E F_{n}(\mathfrak{A}, \mathfrak{B})$ to denote the $n$-move Ehrenfeucht-Fraïssé game on $\mathfrak{A}$ and $\mathfrak{B}$. During each round of the game

\footnotetext{
4 The use of saturated models is problematic because without GCH or inaccessible cardinals we do not know the existence of such. To go around this one can use so-called special models or so called recursively saturated models.
} 
player I first picks an element from one of the models, and then player II picks an element from the other model. In this way a relation

$$
p=\left\{\left(a_{1}, b_{1}\right), \ldots,\left(a_{n}, b_{n}\right)\right\} \subseteq M \times M^{\prime}
$$

is built. If $p$ is a partial isomorphism between $\mathfrak{M}$ and $\mathfrak{M}^{\prime}$, player II is the winner of this play. Player II has a winning strategy in this game if and only if the models $\mathfrak{M}$ and $\mathfrak{M}^{\prime}$ satisfy the same first order sentences of quantifier rank at most $n$. If player II has a winning strategy $\tau$ in $E F_{n}(\mathfrak{A}, \mathfrak{B})$, the set $I_{i}$ of positions

$$
\left\{\left(a_{1}, b_{1}\right), \ldots,\left(a_{j}, b_{j}\right)\right\} \subseteq M \times M^{\prime}
$$

which can be continued to a position

$$
\left\{\left(a_{1}, b_{1}\right), \ldots,\left(a_{n-i}, b_{n-i}\right)\right\} \subseteq M \times M^{\prime}
$$

in which player II has used $\tau$, form an increasing chain $I_{n} \subseteq I_{n-1} \subseteq \ldots \subseteq I_{0}$ known as a back-and-forth sequence and introduced by Fraïssé (1956). The name derives from the fact that if $p \in I_{i+1}$ and $a \in M$ (or $b \in M^{\prime}$ ), then there is $b \in M^{\prime}$ (respectively, $a \in M)$ such that $p \cup\{(a, b)\} \in I_{i}$. Conversely, if a back-and-forth sequence $I_{n} \subseteq I_{n-1} \subseteq \ldots \subseteq I_{0}$ exists, then player II can use it to win the game $E F_{n}(\mathfrak{A}, \mathfrak{B})$. So back-and-forth sequences and winning strategies of II go hand in hand. This explains why the game is generally called the Ehrenfeucht-Fraïssé game.

We can think of the game $E F_{n}(\mathfrak{A}, \mathfrak{B})$ as a restricted case of the simpler game $E F(\mathfrak{A}, \mathfrak{B})$ which lasts for $\omega$ moves. The restriction imposed in $E F_{n}(\mathfrak{A}, \mathfrak{B})$ is that the game is suddenly stopped after $n$ moves. This is as if there was a clock that ticks down, starting from $n$, and going through elements of $n$ in reverse order in $\leq n$ steps. Such a clock can be thought of as a linear order of length $n$. We say that the clock ranks the game.

The following proof of the Robinson property for $L_{\omega \omega}$, using the back-and-forth method just described, is due to Lindström. This is the argument that became the proof of Theorem 1 in Lindström (1969). Let $L_{1}$ be the vocabulary of $T$ and $L_{2}$ that of $T^{\prime}$. By assumption, there is a model $\mathfrak{M}_{1}$ of $T$ and a model $\mathfrak{M}_{2}$ of $T^{\prime}$ such that $\mathfrak{M}_{1}\left\lceil L \equiv \mathfrak{M}_{2}\lceil L\right.$, where $L$ is the common vocabulary. Thus there is, for any $n \in \mathbb{N}$, a back-and-forth sequence $\left(I_{i}: i \leq n\right)$ for $\left.\mathfrak{M}_{1}\right\rceil L$ and $\mathfrak{M}_{2} \uparrow L$. Let $L_{2}^{\prime}$ be a copy of $L_{2}$ such that $L_{2} \cap L_{2}^{\prime}=\emptyset$. Let $L^{\prime}$ be the vocabulary resulting from $L$ in this translation. Let $\mathfrak{M}_{2}^{\prime}$ be the translation of $\mathfrak{M}_{2}$ to the vocabulary $L_{2}^{\prime}$. Let $S$ be the set of first order sentences which state

1. The complete $L_{1}$-theory of $\mathfrak{M}_{1}$,

2. The complete $L_{2}^{\prime}$-theory of $\mathfrak{M}_{2}^{\prime}$

3. $(R,<)$ (the "clock") is a non-empty linear order in which every element with a predecessor has an immediate predecessor.

4. First order sentences which state, by means of new predicates, that there is as a back-and-forth sequence for the $L$-part of the universe and the $L^{\prime}$-part of the universe, ranked by the clock $(R,<)$. 
For all $n \in \mathbb{N}$ there is a model of $S$ with $(R,<)$ of length $n$. By the Compactness Theorem, there is a model $\mathfrak{N}$ of $S$ with $(R,<)$ non-well-founded. By the Löwenheim Property we may assume $\mathfrak{N}$ is countable. Let $\mathfrak{N}_{1}=\mathfrak{N} \uparrow L_{1}$ and $\mathfrak{N}_{2}^{\prime}=\mathfrak{N} \uparrow L_{2}^{\prime}$. Since the clock of $\mathfrak{N}$ is non-well-founded, player II has a winning strategy for the infinite game $E F\left(\mathfrak{N}_{1}, \mathfrak{N}_{2}\right)$, where $\mathfrak{N}_{2}$ is the translation of $\mathfrak{N}_{2}^{\prime}$ to $L_{2}$. Since $\mathfrak{N}_{1}$ and $\mathfrak{N}_{2}$ are countable, $\mathfrak{N}_{1} \cong \mathfrak{N}_{2}$. This implies that $\mathfrak{N}_{1}$ can be expanded to a model of $T \cup T^{\prime}$.

Lindström observed that the above argument works for any logic which has the countable compactness property and the Löwenheim property. But are there such extensions of $L_{\omega \omega}$ ? To see why there are not, let us write $\mathfrak{M} \sim_{n} \mathfrak{M}^{\prime}$ if player II has a winning strategy in $E F_{n}(\mathfrak{A}, \mathfrak{B})$. This equivalence relation divides the class of all models with vocabulary $L$ into a finite number of equivalence classes, each definable by a first order sentence of quantifier rank at most $n$. Thus:

A class of models of a finite relational vocabulary $L$ is first order definable if and only if it is closed under $\sim_{n}$ for some $n \in \mathbb{N}$. (Fraïssé 1956)

Now we can return to Lindström's proof. Let us use the same argument as above to derive the separation property instead of the stronger Robinson property. Suppose $K_{1}$ and $K_{2}$ are disjoint PC-classes of models. We show that there is an EC-class $K$ that separates $K_{1}$ and $K_{2}$, i.e. $K_{1} \subseteq K$ and $K_{2} \cap K=\emptyset$. Case 1: There is an $n \in \mathbb{N}$ such that some union $K$ of $\sim_{n}$-equivalence classes of models separates $K_{1}$ and $K_{2}$. By the above remark, the model class $K$ is first order definable, so the claim is proved. Case 2: There are, for any $n \in \mathbb{N}, L$-models $\mathfrak{M}_{n}$ and $\mathfrak{N}_{n}$ such that $\mathfrak{M}_{n} \sim_{n} \mathfrak{N}_{n}$. Suppose $K_{1}$ is the class of reducts of models of $\varphi$, and $K_{2}$ respectively the class of reducts of models of $\psi$. Let $T$ be the set of first order sentences which state

1. $\varphi^{\left(P_{1}\right)}$.

2. $\psi^{\left(P_{2}\right)}$.

3. $(R,<)$ (the "clock") is a non-empty linear order in which every element with a predecessor has an immediate predecessor.

4. First order sentences which state, by means of new predicates, that there is as a back-and-forth sequence for the $P_{1}$-part of the universe and the $P_{2}$-part of the universe, ranked by the clock $(R,<)$.

For all $n \in \mathbb{N}$ there is a model $\mathfrak{A}_{n}$ of $T$, built from $\mathfrak{M}_{n}$ and $\mathfrak{N}_{n}$, with $(R,<)$ of length $n$. By the Compactness Theorem, there is a countable model $\mathfrak{A}$ of $T$ with $(R,<)$ non-well-founded. Let $\mathfrak{B}_{1}$ be the $L_{1} \cap L_{2}$-structure $\left(\mathfrak{A} \uparrow\left(L_{1} \cap L_{2}\right)\right)^{\left(P_{1}^{\mathfrak{A}}\right)}$. Let $\mathfrak{B}_{2}$ be the $L_{1} \cap L_{2}$-structure $\left(\mathfrak{A} \uparrow\left(L_{1} \cap L_{2}\right)\right)^{\left(P_{2}^{\mathfrak{A}}\right)}$. Now $\mathfrak{B}_{1} \simeq_{p} \mathfrak{B}_{2}$. Since $\mathfrak{B}_{1}$ and $\mathfrak{B}_{2}$ are countable, they are isomorphic. But $\mathfrak{B}_{1} \in K_{1}$ and $\mathfrak{B}_{2} \in K_{2}$, a contradiction.

So we have derived the separation property and the proof clearly works for any logic which satisfies countable compactness and the Löwenheim property. But the conclusion still is that the disjoint $\mathrm{PC}(\mathcal{L})$-classes can be separated by an $\mathrm{EC}\left(L_{\omega \omega}\right)$-class. But then since we assume our logics are closed under negation, every $\operatorname{EC}(\mathcal{L})$-class must be an $\operatorname{EC}\left(L_{\omega \omega}\right)$-class. This is how Lindström came to prove his Theorem 1 . While the theorem itself is famous, it is perhaps less known that the proof, as presented above, gives an alternative proof of the interpolation theorem in first order logic. 


\subsubsection{The diamond of weaker forms of interpolation}

Let us now return to the question whether $L\left(Q_{1}\right)$ or other countably compact logics may satisfy a weaker form of interpolation. A result of Shelah (1985) says that there is a compact extension of first order logic with Beth. The logic is the smallest extension of a close relative of $L\left(Q_{\omega}^{\text {cof }}\right)$ to a logic with the Beth property. The proof extends the proof of the compactness of the base logic, step by step, to the whole Beth extension. There is also a compact logic with the Souslin-Kleene interpolation property, namely $\Delta\left(L\left(Q_{\omega}^{\text {cof }}\right)\right)$. Here all we need to know is that $L\left(Q_{\omega}^{\text {cof }}\right)$ is compact as $\Delta$ always preserves compactness. Surprisingly, considering the difficulty in finding any positive results about interpolation among generalized quantifier logics, Mekler and Shelah (1985) showed that it is consistent, relative to the consistency of ZFC, that $L\left(Q_{1}\right)$ satisfies the weak Beth property. It is still not known whether this is in fact provable in ZFC. The proof of Mekler and Shelah is based on the idea that we kill by forcing all implicit definitions which do not have an explicit definition. Although this may sound ad hoc, the proof actually gives a useful criterion, presented in Shelah (1985), for a countably compact logic to satisfy the weak Beth property. The full extent of this idea has not been exhausted yet.

\section{The infinitary trail}

The infinitary trail extends from first order logic, through the "pseudo" infinitary logic $L\left(Q_{0}\right)$ to two main branches, the lower branch of classical infinitary languages $L_{\kappa \omega}$, and further on $L_{\kappa \kappa}$, and the upper branch of variants with the game quantifier (or just the well-ordering quantifier $Q^{\text {wo }}$ ). These two main branches are radically different from the point of view of interpolation. On the lower branch we get results up to the point where well-ordering becomes definable, while on the upper branch well-ordering is definable right in the beginning, and negative results start pouring in.

\subsection{Negative results}

A sentence $\varphi$ of a logic $\mathcal{L}$ is said to pin down the ordinal $\alpha$ if $\tau(\varphi)$ has a binary predicate $<$ such that in every model $\mathfrak{A}$ of $\varphi$ we have $<{ }^{\mathfrak{A}}$ well-ordered, and additionally, in some model $\mathfrak{A}$ of $\varphi$ we have $<^{\mathfrak{A}}$ well-ordered in type $\geq \alpha$. First order logic and any countably compact logic can pin down finite ordinals only. The logic $L_{\kappa \omega}$ pins down $\kappa^{+}$if $\kappa$ is regular (Barwise and Kunen 1971), and even $2^{\kappa}$ if $\kappa$ is a singular strong limit (Shelah 1990). The question how large ordinals a logic pins down is a vitally important characteristic of the logic related, e.g. to the interpolation property, models with indiscernibles, and Hanf numbers. For interpolation the relevant relationship was established by Gostanian and Hrbáček (1979): If $L_{\kappa+\omega}$ pins down a regular $\lambda$, then Craig $\left(L_{\kappa^{+} \omega}, L_{\lambda \lambda}\right)$ fails. Combined with (5) below, this shows that no sentence of $L_{\infty \omega}$ pins down every ordinal.

The proof of Malitz (1971) that $L_{\infty \omega}$ does not have the interpolation property can be used to show: If $\mathcal{L} \leq \mathcal{L}^{\prime}, \mathcal{L}$ has a consistent sentence $\varphi$ with only countable models and a consistent sentence $\psi$ with only uncountable models, and $\operatorname{Craig}\left(\mathcal{L}, \mathcal{L}^{\prime}\right)$ holds, 
then $\mathcal{L}^{\prime}$ cannot have the Karp property. ${ }^{5}$ The reason is simple: We may assume that the vocabularies of $\varphi$ and $\psi$ are disjoint. So $\models \varphi \rightarrow \neg \psi$. However, all infinite models of the empty vocabulary are partially isomorphic. So no sentence of the empty vocabulary in a logic with the Karp property can separate $\varphi$ and $\neg \psi$. Barwise formulated this as follows: If an abstract logic has the many-sorted interpolation property, then it has the Karp property if and only if it has the Löwenheim property. As a consequence, no logic between $L_{\omega_{2} \omega}$ and $L_{\infty G}$ can have the (many-sorted) interpolation property, as they all have the Karp property. Moreover, no absolute logic $\mathcal{L}$ can satisfy $\operatorname{Craig}\left(L_{\omega_{2} \omega}, \mathcal{L}\right)$, again for the same reason.

One can also discern a purely mathematical impediment for the interpolation property in logics based on uncountable structres, such as $L_{\omega_{2} \omega}$ and its extensions: Let us consider $\omega_{1}^{\omega_{1}}$ as a generalized Baire space with the so called box topology. The family of Borel sets is the smallest set containing open sets and closed under complements and unions of length $\omega_{1}$. We can consider classes of models of size $\aleph_{1}$ as subsets in this space. Assume $\mathrm{CH}$. Then there are disjoint $\Sigma_{1}^{1}$ sets which cannot be separated by a Borel set. Assume, on the other hand, not-CH+MA. Then any two disjoint $\Sigma_{1}^{1}$ sets of expansions of $\left(\omega_{1},<\right)$ can be separated by a Borel set (Shelah and Väänänen 2000).

\subsection{Positive results}

The proof theoretic argument for the interpolation property of $L_{\omega \omega}$, proceeding via the Cut Elimination Theorem of a suitable Gentzen system, works also in $L_{\omega_{1} \omega}$, as was demonstrated by Lopez-Escobar (1965). It works also in the countable admissible fragments of $L_{\omega_{1} \omega}$, as demonstrated by Barwise (1969). The best result in this direction in the classical higher infinitary logics is the following result of Malitz (1971):

$$
\operatorname{Craig}\left(L_{\kappa \omega}, L_{\lambda \kappa}\right) \text {, where } \lambda=\left(2^{<\kappa}\right)^{+} \text {and } \kappa \text { is regular. }
$$

The proof of interpolation by means of the Robinson property has the obvious stumbling block that infinitary logics are not countably compact. However, the Lindström variant works to some extent as was shown by Barwise (1974). A little surprisingly, we do not get a proof of interpolation but merely a model theoretic characterization of the infinitary logic $L_{\kappa}+\omega$ : Suppose $\kappa=\beth_{\kappa}$. Then $L_{\kappa \omega}$ is the maximal extension of $L_{\omega \omega}$ which has the Karp property and that pins down only ordinals $<\kappa$. The proof is an adaption of the proof of Lindström's Theorem.

\subsection{Consistency properties}

Henkin (1963) gave a proof of interpolation for $L_{\omega \omega}$ which is based on a semantic concept of proof. The idea of Henkin's proof is the following: Let $T_{1}$ be a theory in the vocabulary $L_{1}$, and $T_{2}$ a theory in the vocabulary $L_{2}$. Let us say that a sentence $\theta$ in the vocabulary $L=L_{1} \cap L_{2}$ separates the theories $T_{1}$ and $T_{2}$, making them separable, if $T_{1} \models \theta$ and $T_{2} \models \neg \theta$. Starting from the assumption that the theories $\{\varphi\}$ and $\{\neg \psi\}$ are

\footnotetext{
5 Any two partially isomorphic structures are elementarily equivalent
} 
inseparable, Henkin builds step by step a theory $S$ such that $\{\varphi, \neg \psi\} \subseteq S$. Two special parts of $S$, consisting solely of sentences of the vocabulary $L_{1}$, and respectively $L_{2}$, are kept inseparable in the process, and in addition $S$ satisfies conditions familiar from his proof of Gödel's Completeness Theorem. The latter conditions guarantee that we can build a "Henkin"-model for $S$. But this contradicts the assumption that $\models \varphi \rightarrow \psi$. So the original assumption that $\{\varphi\}$ and $\{\neg \psi\}$ are inseparable must be abandoned.

Henkin's proof was elaborated and applied to $L_{\omega_{1} \omega}$ in Makkai (1969). He introduced the method of consistency properties to infinitary logic and used it to prove interpolation and preservation results. By elaborating further, a proof of (5) can be given by means of consistency properties.

\subsection{Approximation of game expressions}

Svenonius (1965) gave a game theoretic proof of interpolation for $L_{\omega \omega}$ anticipating the emergence of the Covering Theorem of descriptive set theory in infinitary logic. Svenonius associates with a given $\operatorname{PC}\left(L_{\omega \omega}\right)$ class $K$ a game sentence $\Phi$ :

$$
\forall x_{0} \exists x_{1}\left(\varphi_{0}\left(x_{0}, x_{1}\right) \wedge \forall x_{2} \exists x_{3}\left(\varphi_{1}\left(x_{0}, x_{1}, x_{2}, x_{3}\right) \wedge \ldots\right)\right)
$$

which is true in every model in $K$. Conversely, every countable model of $\Phi$ is in $K$. The intuition is the following. Suppose, for simplicity, that the $\operatorname{PC}\left(L_{\omega \omega}\right)$ definition of $K$ is

$$
\exists f \forall x_{0} \exists x_{1} \varphi\left(x_{0}, x_{1}, f\left(x_{0}\right), f\left(x_{1}\right)\right),
$$

where $\varphi\left(x_{0}, x_{1}, y_{0}, y_{1}\right)$ is quantifier free. The formula (6) would in this case be

$$
\begin{aligned}
& \forall x_{0} \forall x_{1} \exists y_{0} \exists y_{1}\left(\varphi\left(x_{0}, x_{1}, y_{0}, y_{1}\right) \wedge\right. \\
& \forall x_{2} \forall x_{3} \exists y_{2} \exists y_{3}\left(\varphi\left(x_{2}, x_{3}, y_{2}, y_{3}\right) \wedge\right. \\
& \left(x_{0}=x_{2} \rightarrow y_{0}=y_{2}\right) \wedge\left(x_{1}=x_{3} \rightarrow y_{1}=y_{3}\right) \wedge \\
& \ldots))
\end{aligned}
$$

To prove the equivalence, suppose first

$$
(\mathfrak{A}, f) \models \forall x_{0} \exists x_{1} \varphi\left(x_{0}, x_{1}, f\left(x_{0}\right), f\left(x_{1}\right)\right) .
$$

The winning strategy of the player who makes the choices for each $\exists y_{i}$ in (8) is to always play $y_{i}=f\left(x_{i}\right)$. On the other hand, if $A=\left\{a_{0}, a_{1}, \ldots\right\}$ and (8) holds in $\mathfrak{A}$, then the responses $y_{i}=b_{i}$ to the assignment $x_{i}=a_{i}$ can be used to define a function $f\left(a_{i}\right)=b_{i}$ such that (9) holds. The next step in the proof by Svenonius is to form the following approximations ${ }^{6}$ of (8):

\footnotetext{
6 Such approximations were also considered by Keisler (1965).
} 


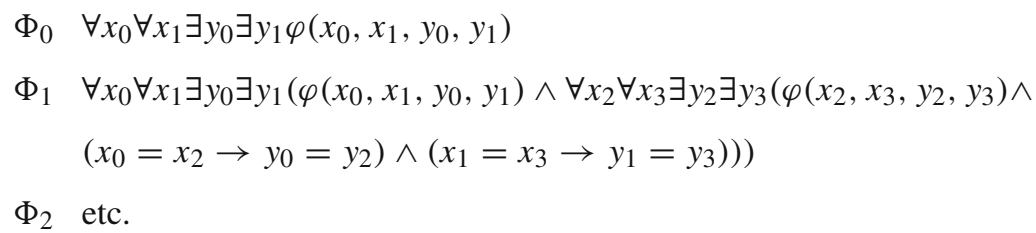

Clearly, (8) implies each of its approximations $\Phi_{n}$. Conversely, it is easy to see that if a saturated ${ }^{7}$ model satisfies each $\Phi_{n}$, it satisfies the whole (8). The proof of the interpolation property for $L_{\omega \omega}$ proceeds now as follows: Suppose $K$ and $K^{\prime}$ are two disjoint $\mathrm{PC}\left(L_{\omega \omega}\right)$ classes. Let $\Phi$ and $\Psi$ be the corresponding game formulas. If the theory

$$
\left\{\Phi_{n}: n<\omega\right\} \cup\left\{\Psi_{n}: n<\omega\right\}
$$

is consistent, it has a saturated model $\mathfrak{A}$. But then $\mathfrak{A}$ is in $K \cap K^{\prime}$ which we assumed to be empty. So the theory (10) is inconsistent and some $\Phi_{n}$ separates the model classes. A nice feature of this proof is that the syntax of the interpolant has a close resemblance to the syntax of the starting sentences $\varphi$ and $\psi$. In fact, the Svenonius approach has been used in a variety of ways in definability theory and the theory of preservation theorems. What is even more interesting, at least from the point of view of abstract model theory, is that the Svenonius approach lends itself directly to infinitary logic. In infinitary logic we cannot use saturated models, because we do not have compactness, but the appeal to saturated models is actually an overkill. The same argument can be realized in a more delicate way by means of information about upper bounds for "pinning down" ordinals. Makkai has a good survey of this approach in Barwise et al. (1977).

Let us now see how the method of approximations of game expressions works in some detail in infinitary logic. Vaught (1973) associates a given $\operatorname{PC}\left(L_{\omega_{1} \omega}\right)$ class $K$ of vocabulary $L$ with the game formula:

$$
\begin{gathered}
\forall x_{0} \bigvee_{a_{0} \in \omega} \bigwedge_{a_{1} \in \omega} \exists x_{1}\left(\varphi_{\left(a_{0}, a_{1}\right)}^{0}\left(x_{0}, x_{1}\right) \wedge\right. \\
\forall x_{2} \bigvee_{a_{2} \in \omega} \bigwedge_{a_{3} \in \omega} \exists x_{3}\left(\varphi_{\left(a_{0}, a_{1}, a_{2}, a_{3}\right)}^{1}\left(x_{0}, x_{1}, x_{2}, x_{3}\right) \wedge\right. \\
\ldots \\
\forall x_{2 n} \bigvee_{a_{2 n} \in \omega} \bigwedge_{a_{2 n+1} \in \omega} \exists x_{2 n+1}\left(\varphi_{\left(a_{0}, \ldots, a_{2 n+1}\right)}^{n}\left(x_{0}, \ldots, x_{2 n+1}\right) \wedge\right. \\
\ldots) \ldots),
\end{gathered}
$$

where $\varphi_{\left(a_{0} \ldots a_{2 n+1}\right)}\left(x_{0}, \ldots, x_{2 n+1}\right)$ is a conjunction of atomic and negated atomic formulas in vocabulary $L$ in the variables $x_{0}, \ldots, x_{2 n+1}$. The sentence (11) is designed so that if $\mathfrak{A} \in K$, then the player playing $\exists$ and $\bigvee$ in (11) has an easy winning strategy.

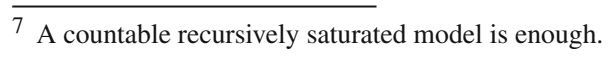


On the other hand, if a countable model $\mathfrak{B}$ satisfies (11), then $\mathfrak{B} \in K$, essentially because partially isomorphic countable models are isomorphic.

Let us consider the following approximations of (11):

$$
\Phi_{\left(a_{0}, \ldots, a_{2 n-1}\right)}^{0}\left(x_{0}, \ldots, x_{2 n-1}\right)
$$

is the conjunction of $\Phi_{\left(a_{0}, \ldots, a_{2 m+1}\right)}^{m}\left(x_{0}, \ldots, x_{2 m+1}\right)$ for $m$

$$
\Phi_{\left(a_{0}, \ldots, a_{2 n-1}\right)}^{\alpha+1}\left(x_{0}, \ldots, x_{2 n-1}\right)
$$

is

$$
\forall x_{2 n} \bigvee_{a_{2 n} \in \omega} \bigwedge_{a_{2 n+1} \in \omega} \exists x_{2 n+1} \Phi_{\left(a_{0}, \ldots, a_{2 n+1}\right)}^{\alpha}\left(x_{0}, \ldots, x_{2 n+1}\right),
$$

and for limit $v$

$$
\Phi_{\left(a_{0}, \ldots, a_{2 n-1}\right)}^{v}\left(x_{0}, \ldots, x_{2 n-1}\right)=\bigwedge_{\alpha<v} \Phi_{\left(a_{0}, \ldots, a_{2 n-1}\right)}^{\alpha}\left(x_{0}, \ldots, x_{2 n-1}\right) .
$$

Note that unlike (11), the formulas $\Phi_{\left(a_{0}, \ldots, a_{2 n-1}\right)}^{\alpha+1}\left(x_{0}, \ldots, x_{2 n-1}\right)$ are all in $L_{\infty \omega}$. We write $\Phi^{\alpha}$ for $\Phi_{()}^{\alpha}$. Clearly, if player II has a winning strategy in (11) in a model $\mathfrak{B}$, then $\mathfrak{B} \models \Phi^{\alpha}$ for all $\alpha$. On the other hand, a simple cardinality argument shows that if a model of size $\kappa$ satisfies $\Phi^{\alpha}$ for all $\alpha<\kappa$, then the model satisfies (11).

Thus if we have two disjoint $\mathrm{PC}\left(L_{\omega_{1} \omega}\right)$ classes $K_{1}$ and $K_{2}$, we can associate with them two conjunctive game expressions $\Phi_{1}$ and $\Phi_{2}$ such that $\Phi_{1}$ and $\Phi_{2}$ have no countable models in common. Let $\Phi_{1}^{\alpha}$ and $\Phi_{2}^{\alpha}$ be the approximations of $\Phi_{1}$ and $\Phi_{2}$, respectively. There must be an $\alpha<\omega_{1}$ such that $\Phi_{1}^{\alpha}$ and $\Phi_{2}^{\alpha}$ have no models in common, for otherwise we can pin down every countable ordinal, a contradiction with Lindström (1966b) and Lopez-Escobar (1966). Now $\Phi_{1}^{\alpha}$ is the desired interpolant. The argument used is called the Covering Theorem because the approximations "cover" the game formula.

Makkai (1971) gives a unified treatment of preservation theorems in a generalized framework using approximations of game expressions. Harnik and Makkai (1976) further give applications of the Covering Theorem to model theory using Vaught formulas. Makkai (1974) generalized the covering theorem approach to $L_{\kappa} \omega, \kappa$ of strong cofinality $\omega$.

\subsection{Between $L_{\kappa \omega}$ and $L_{\kappa \kappa}$ for $\kappa>\omega_{1}$}

Recall the relative interpolation theorem (5) of Malitz. For strongly inaccessible $\kappa$ this yields Craig $\left(L_{\kappa \omega}, L_{\kappa \kappa}\right)$. Of course neither Craig $\left(L_{\kappa \omega}, L_{\kappa \omega}\right)$, nor $\operatorname{Craig}\left(L_{\kappa \kappa}, L_{\kappa \kappa}\right)$ hold. But is there an intermediate logic $\mathcal{L}$ between $L_{\kappa \omega}$ and $L_{\kappa \kappa}$ for strongly inaccessi- 
ble $\kappa$ ? Indeed, such $\mathcal{L}$ exists, as demonstrated in Shelah and Väänänen (to appear). To simplify notation, let us redefine $L_{\kappa \omega}$ as $\bigcup_{\lambda<\kappa} L_{\lambda \omega}$ and $L_{\kappa \kappa}$ as $\bigcup_{\lambda<\kappa} L_{\lambda \lambda}$ in the case that $\kappa$ is a limit cardinal. For regular limits this agrees with the old notation for $L_{\kappa \omega}$ and $L_{\kappa \kappa}$, but for singular cardinals the new notation seems canonical. Let $\kappa=\beth_{\kappa}$. The new infinitary logic $L_{\kappa}^{+}$introduced in Shelah and Väänänen (to appear) satisfies $L_{\kappa \omega} \leq L_{\kappa}^{+}, L_{\kappa}^{+} \leq L_{\kappa \kappa}$ and $\operatorname{Craig}\left(L_{\kappa}^{+}\right)$. Moreover, $L_{\kappa}^{+}$has a Lindström style model theoretic characterization in terms of a strong form of undefinability of well-order. The main ingredient of $L_{\kappa}^{+}$is a new variant of the Ehrenfeucht-Fraïssé game for $L_{\kappa \kappa}$. In the new variant player II gives only partial answers to moves of player I. If the game lasted for $\omega$ moves, the partial answers of II would constitute full answers. But the game has a well-founded clock, so II never ends up fully completing her answers. Although $L_{\kappa}^{+}$cannot express well-ordering, it can express the property of a linear order of not having uncountable descending chains.

\subsection{Going beyond $L_{\kappa \kappa}$}

There are, as we have seen above, strong negative results about interpolation for logics extending $L_{\omega_{1} \omega_{1}}$, indeed for just any logic, like $L_{\omega_{1} G}$, capable of expressing well-ordering. Even $L_{\infty \infty}$ is not enough. However, the Henkin proof of interpolation for first order logic, generalized to $L_{\omega_{1} \omega}$ by Makkai, can be adapted, as shown by Hyttinen (1990) and Tuuri (1992), to give proofs of separation theorems in large infinitary logics. The central concept is the concept of a transfinite Model Existence Game and the concept of a transfinite Ehrenfeucht-Fraïssé game.

So what needs to be added to $L_{\infty \infty}$ in order that interpolation starts to work? One important ingredient of the proof according to Henkin and Svenonius was that countable partially isomorphic structures are isomorphic. When we proceed to higher infinitary logics we have to handle uncountable structures. As observed by Tait and Morley already in the sixties, uncountable $L_{\infty \kappa}$-equivalent models of cardinality $\kappa>\omega$ need not be isomorphic. The situation changes if the EhrenfeuchtFraïssé game of $L_{\infty \kappa}$ is continued for $\kappa$ moves. This solves the problem observed by Tait and Morley. However, a new problem emerges: this new game need not be determined (Mekler et al. 1993). However, let us overlook this problem for a moment and work towards a proof of the separation property, where negation is not needed.

We assume $\kappa=\kappa^{<\kappa}$ below. It is possible to extend both the proof according to Henkin, based on consistency properties, and the proof according to Svenonius and Vaught, based on approximations of game formulas, to uncountable models by systematically employing games longer than $\omega$. When approximations of such games are built, we need to use clocks that are non-well-founded. A natural choice is to use trees as clocks. Time passes step by step up the branches of the tree until the branch ends. A well-founded clock corresponds to a tree with no infinite branches. When such trees are used to measure the length of a game, and approximations of the relevant game formulas are formed, the approximations are not necessarily formulas of $L_{\infty \infty}$. This is where $M_{\kappa}{ }^{+}{ }_{\kappa}$ steps into the picture. The formulas of $M_{\kappa^{+}{ }_{\kappa}}$ are locally like formulas 
of $L_{\kappa}{ }^{+}{ }_{\kappa}$ but descending subformula sequences can be transfinite. ${ }^{8}$ With this extension of $L_{\kappa+}{ }_{\kappa}$ we get $\operatorname{Sep}\left(M_{\kappa^{+}{ }_{\kappa}}\right)$ (Hyttinen 1990, Tuuri 1992).

A result of the non-determinedness of some relevant transfinite games is that $M_{\kappa^{+}}{ }_{\kappa}$ is not closed under negation. If one takes the fragment of $M_{\kappa}+{ }_{\kappa}$ consisting of sentences with negation in $M_{\kappa^{+}}$, the so called determined part of $M_{\kappa^{+}{ }_{\kappa}}$, the resulting logic is exactly $\Delta\left(L_{\kappa^{+}}\right)$. It is consistent, relative to the consistency of ZFC, that Craig $\left(L_{\kappa^{+}{ }_{\kappa}}, \Delta\left(L_{\kappa^{+}{ }_{\kappa}}\right)\right)$ fails. So we have found the separation property among the higher infinitary logics, but at the cost of losing full negation and having had to assume $\kappa=\kappa^{<\kappa}$. By means of large cardinals and definability assumptions it may be possible to go around the problem of negation. How to go around the assumption $\kappa=\kappa^{<\kappa}$ is not clear but suitable forcing axioms (like PFA) may play a role.

Just as undefinability of well-order turned out to be a crucial element in the proof of interpolation for the logic $L_{\omega_{1} \omega}$, the undefinability of $\kappa$-well-order (linear order with no descending chains of length $\kappa$, Oikkonen 1997) turns out to be the deciding factor for $\operatorname{Sep}\left(M_{\kappa^{+}{ }_{\kappa}}\right)$.

No maximality results are known for the infinitely deep languages $M_{\kappa^{+}}{ }_{\kappa}$, although such probably can be proved with appropriate set theoretical assumptions.

\section{The higher order realm}

When we come to second order logic it becomes vitally important to make a clear difference between the single-sorted interpolation property and the many-sorted one. In the language of separation properties the corresponding distinction is between PC classes and RPC classes. Second order logic satisfies the single-sorted interpolation property for the trivial reason that $L^{2}=\mathrm{PC}\left(L^{2}\right)$. With a little bit more work one can show that also the Lyndon Theorem holds for $L^{2}$. As to the many-sorted interpolation, Craig proved in Craig (1965) with an undefinability of truth argument that $L^{2}$ fails to satisfy it. This proof became a paradigmatic example of the failure of interpolation and its weaker forms in logics with enough expressive power to characterize their syntax set (for a survey of such results, see Väänänen 1985). In the strange world of higher order logics single-sorted interpolation holds in the strongest possible sense, and the many-sorted interpolation fails in a most manifest way. In the case of all other logics it seems that the single-sorted/many-sorted distinction makes no difference: either both hold for the same reason or both fail for the same reason.

Our claim was that the Craig Interpolation Theorem is connected to maximality results for logic. The maximality characterization of $L^{2}$, arising from the fact $L^{2}=\mathrm{PC}\left(L^{2}\right)$ that lies behind the interpolation property of $L^{2}$, is the following: $L^{2}$ is the maximal extension of $L_{\omega \omega}$ in which every definable model class has a flat definition in set theory (Väänänen 1979).

Acknowledgements Research partially supported by grant 40734 of the Academy of Finland.

\footnotetext{
8 The relevance of such logics was suggested already in Hintikka and Rantala (1976).
} 
Open Access This article is distributed under the terms of the Creative Commons Attribution Noncommercial License which permits any noncommercial use, distribution, and reproduction in any medium, provided the original author(s) and source are credited.

\section{Appendix}

\begin{tabular}{|c|c|c|c|}
\hline Symbol & Name & References & Craig \\
\hline$L_{\omega \omega}$ & First order logic & & $\sqrt{ }$ \\
\hline$L\left(Q_{0}\right)$ & The quantifier "there exists infinitely many" & Mostowski (1957) & \\
\hline$L_{\omega_{1} \omega}$ & $\begin{array}{l}\text { Infinitary logic with countable } \\
\text { conjunctions and disjunction }\end{array}$ & Tarski (1958), Keisler (1971) & $\sqrt{ }$ \\
\hline$L_{\mathrm{HYP}}$ & The smallest admissible fragment & Barwise $(1969,1975)$ & $\sqrt{ }$ \\
\hline$L_{A}$ & The admissible fragment & Barwise $(1969,1975)$ & $\sqrt{ }$ \\
\hline$L\left(Q^{\text {wo }}\right)$ & The well ordering quantifier & Lindström (1966a) & \\
\hline$L_{\omega_{1} G}$ & The game quantifier & Henkin (1959), Keisler (1965) & \\
\hline$L_{\kappa \omega}$ & $\begin{array}{l}\text { Infinitary logic with uncountable } \\
\text { conjunctions and disjunctions }\end{array}$ & Karp (1964) & \\
\hline$L_{\kappa G}$ & Infinitary logic with the game quantifier & Vaught (1973) & \\
\hline$L_{\kappa}^{+}$ & A new infinitary logic & & $\sqrt{ }$ \\
\hline$L_{\kappa \kappa}$ & Infinite quantifier logic & Dickmann (1975) & \\
\hline$M_{\kappa}+{ }_{\kappa}$ & Infinitely deep logic & Hyttinen (1990), Tuuri (1992) & $\sqrt{ }^{*}$ \\
\hline$L\left(Q_{1}\right)$ & $\begin{array}{l}\text { The quantifier "there exists } \\
\text { uncountably many" }\end{array}$ & Mostowski (1957), Keisler (1970) & \\
\hline$L(I)$ & The Härtig quantifier & Härtig (1962), Herre et al. (1991) & \\
\hline$L(a a)$ & The stationary logic & Shelah (1975), Barwise et al. (1978) & \\
\hline$L(H)$ & The Henkin quantifier & Henkin (1959) & \\
\hline$L\left(Q_{\omega}^{\mathrm{cof}}\right)$ & The cofinality quantifier & Shelah (1975) & \\
\hline$L\left(Q_{1}^{\mathrm{MM}}\right)$ & The Magidor-Malitz quantifier & Magidor and Malitz (1977) & \\
\hline$L^{2}$ & Second order logic & & $\sqrt{ }^{* *}$ \\
\hline
\end{tabular}

* Separation, ** Single-sorted

\section{References}

Addison, J. W. (1962). Some problems in hierarchy theory. In Proceedings of Symposia in Pure Mathematics (Vol. V, pp. 123-130). Providence, RI: American Mathematical Society.

Addison, J. W. (2004). Tarski's theory of definability: Common themes in descriptive set theory, recursive function theory, classical pure logic, and finite-universe logic. Annals of Pure and Applied Logic, 126(1-3):77-92. Provinces of logic determined.

Alechina, N., \& van Lambalgen, M. (1996). Generalized quantification as substructural logic. Journal of Symbolic Logic, 61(3), 1006-1044.

Barwise, J. (1969). Infinitary logic and admissible sets. Journal of Symbolic Logic, 34(2), 226-252.

Barwise, J. (1972). Absolute logics and $L_{\infty \omega}$. Annals of Mathematical Logic, 4, 309-340.

Barwise, J. (1974). Axioms for abstract model theory. Annals of Mathematical Logic, 7, 221-265.

Barwise, J. (1975). Admissible sets and structures. An approach to definability theory, Perspectives in mathematical logic. Berlin: Springer-Verlag.

Barwise, J., \& Feferman, S. (Eds.). (1985). Model-theoretic logics. Perspectives in mathematical logic. New York: Springer-Verlag.

Barwise, J., Kaufmann, M., \& Makkai, M. (1978). Stationary logic. Annals of Mathematical Logic, 13(2), $171-224$. 
Barwise, J., Keisler, H. J., Eklof, P. C., Macintyre, A., Morley, M., Stroyan, K. D., Makkai, M., Kock, A., \& Reyes, G. E. (1977). Model theory. In Handbook of mathematical logic, part A. Studies in logic and the foundations of mathematics (Vol. 90, pp. 3-313). Amsterdam: North-Holland.

Barwise, J., \& Kunen, K. (1971). Hanf numbers for fragments of $L_{\infty \omega}$. Israel Journal of Mathematics, 10, $306-320$.

Burgess, J. P. (1977). Descriptive set theory and infinitary languages. Zb. Rad. Mat. Inst. Beograd (N.S.), 2(10), 9-30. Set theory, foundations of mathematics (Proc. Sympos., Belgrade).

Caicedo, X. (1983). Failure of interpolation for quantifiers of monadic type. In Methods in mathematical logic (Caracas, 1983) Lecture Notes in Mathematics (Vol. 1130, pp. 1-12). Berlin: Springer, 1985.

Craig, W. (1957a). Linear reasoning. A new form of the Herbrand-Gentzen theorem. Journal of Symbolic Logic, 22, 250-268.

Craig, W. (1957b). Three uses of the Herbrand-Gentzen theorem in relating model theory and proof theory. Journal of Symbolic Logic, 22, 269-285.

Craig, W. (1965). Satisfaction for $n$-th order languages defined in $n$-th order languages. Journal of Symbolic Logic, 30, 13-25.

Dickmann, M. A. (1975). Large infinitary languages. Model theory, Studies in logic and the foundations of mathematics (Vol. 83). Amsterdam: North-Holland Publishing Co.

Ehrenfeucht, A. (1960/1961). An application of games to the completeness problem for formalized theories. Fundamenta Mathematicae, 49, 129-141.

Feferman, S. (1975). Two notes on abstract model theory. II. Languages for which the set of valid sentences is semi-invariantly implicitly definable. Fundamenta Mathematicae, 89(2), 111-130.

Flum, J. (1985). Characterizing logics. In Model-theoretic logics. Perspectives in mathematical logic (pp. 77-120). New York: Springer.

Fraïssé, R. (1956). Sur quelques classifications des relations, basées sur des isomorphismes restreints. Publ. Sci. Univ. Alger. Sér. A, 2, 15-60, 1955.

Friedman, H. (1973). Beth's theorem in cardinality logics. Israel Journal of Mathematics, 14, $205-212$.

Gostanian, R., \& Hrbáček, K. (1976). On the failure of the weak Beth property. Proceedings of the American Mathematical Society, 58, 245-249.

Harnik, V., \& Makkai, M. (1976). Applications of Vaught sentences and the covering theorem. Journal of Symbolic Logic, 41(1), 171-187.

Härtig, K. (1962). Über einen Quantifikator mit zwei Wirkungsbereichen. In Colloq. Found. Math., Math. Machines and Appl. (Tihany, 1962) (pp. 31-36). Budapest: Akad. Kiadó, 1965.

Henkin, L. (1959). Some remarks on infinitely long formulas. In Infinitistic Methods (Proc. Sympos. Foundations of Math., Warsaw) (pp. 167-183). Oxford: Pergamon, 1961.

Henkin, L. (1963). An extension of the Craig-Lyndon interpolation theorem. Journal of Symbolic Logic, 28, 201-216.

Herre, H., Krynicki, M., Pinus, A., \& Väänänen, J. (1991). The Härtig quantifier: A survey. Journal of Symbolic Logic, 56(4), 1153-1183.

Hintikka, J., \& Rantala, V. (1976). A new approach to infinitary languages. Annals of Mathematical Logic, 10(1), 95-115.

Hyttinen, T. (1990). Model theory for infinite quantifier languages. Fundamenta Mathematicae, 134(2), $125-142$.

Karp, C. R. (1964). Languages with expressions of infinite length. Amsterdam: North-Holland Publishing Co.

Keisler, H. J. (1961). Ultraproducts and elementary classes. Nederl. Akad. Wetensch. Proc. Ser. A $64=$ Indag. Math., 23, 477-495.

Keisler, H. J. (1965). Finite approximations of infinitely long formulas. In Theory of models (Proceedings of 1963 International Symposium, Berkeley) (pp. 158-169). Amsterdam: North-Holland.

Keisler, H. J. (1970). Logic with the quantifier "there exist uncountably many". Annals of Mathematical Logic, 1, 1-93.

Keisler, H. J. (1971). Model theory for infinitary logic. Logic with countable conjunctions and finite quantifiers. Studies in logic and the foundations of mathematics (Vol. 62). Amsterdam: North-Holland Publishing Co.

Lindström, P. (1966a) First order predicate logic with generalized quantifiers. Theoria, 32, 186-195.

Lindström, P. (1966b). On characterizability in L $\omega_{1} \omega_{0}$. Theoria, 32, 165-171.

Lindström, P. (1969). On extensions of elementary logic. Theoria, 35, 1-11. 
Lindström, P. (1995). Prologue. In M. Krynicki, M. Mostowski, \& L. W. Szczerba (Eds.), Quantifiers: Logics, models and computation: Surveys v. 1 (pp. 21-24). Dordrecht: Kluwer Academic Publishers.

Lopez-Escobar, E. G. K. (1965). An interpolation theorem for denumerably long formulas. Fundamenta Mathematicae, 57, 253-272.

Lopez-Escobar, E. G. K. (1966). On defining well-orderings. Fundamenta Mathematicae, 59, 13-21.

Magidor, M., \& Malitz, J. (1977). Compact extensions of L(Q). Ia. Annals Mathematical Logic, 11(2), 217-261.

Makkai, M. (1969). On the model theory of denumerably long formulas with finite strings of quantifiers. Journal of Symbolic Logic, 34, 437-459.

Makkai, M. (1971) Svenonius sentences and Lindström's theory on preservation theorems. Fundamenta Mathematicae, 73(3), 219-233, 1971/72.

Makkai, M. (1974). Generalizing Vaught sentences from $\omega$ to strong confinality $\omega$. Fundamenta Mathematicae, 82, 105-119. Collection of articles dedicated to Andrzej Mostowski on the occasion of his sixtieth birthday, VI.

Makowsky, J. A., \& Shelah, S. (1983). Positive results in abstract model theory: A theory of compact logics. Annals of Pure and Applied Logic, 25(3), 263-299.

Malitz, J. (1971). Infinitary analogs of theorems from first order model theory. Journal of Symbolic Logic, 36, 216-228.

Mekler, A., \& Shelah, S. (1985). Stationary logic and its friends. I. Notre Dame Journal of Formal Logic, 26(2), 129-138.

Mekler, A., Shelah, S., \& Väänänen, J. (1993) The Ehrenfeucht-Fraïssé-game of length $\omega_{1}$. Transactions of the American Mathematical Society, 339(2), 567-580.

Mostowski, A. (1957). On a generalization of quantifiers. Fundamenta Mathematicae, 44, 12-36.

Mostowski, A. (1968) Craig's interpolation theorem in some extended systems of logic. In Logic, methodology and philosophical science III (Proceedings of Third International Congress, Amsterdam, 1967) (pp. 87-103). Amsterdam: North-Holland.

Oikkonen, J. (1997). Undefinability of $\kappa$-well-orderings in $L_{\infty \kappa}$. Journal of Symbolic Logic, 62(3), 9991020.

Shelah, S. (1975). Generalized quantifiers and compact logic. Transactions of the American Mathematical Society, 204, 342-364.

Shelah, S. (1985). Remarks in abstract model theory. Annals of Pure and Applied Logic, 29(3), 255-288.

Shelah, S. (1990). Classification theory and the number of nonisomorphic models. Volume 92 of Studies in logic and the foundations of mathematics (2nd ed.). Amsterdam: North-Holland Publishing Co.

Shelah, S., \& Väänänen, J. New infinitary languages with interpolation (to appear).

Shelah, S., \& Väänänen, J. (2000). Stationary sets and infinitary logic. Journal of Symbolic Logic, 65(3), 1311-1320.

Svenonius, L. (1965). On the denumerable models of theories with extra predicates. In Theory of models (Proceedings of 1963 International Symposium, Berkeley) (pp 376-389). Amsterdam: North-Holland.

Szabo, M. E. (1987). A cut elimination theorem for stationary logic. Annals of Pure and Applied Logic, 33(2), 181-193.

Tarski, A. (1958). Remarks on predicate logic with infinitely long expressions. Colloquium Mathematicum, $6,171-176$

Tuuri, H. (1992). Relative separation theorems for $L_{\kappa+\kappa}$. Notre Dame Journal of Formal Logic, 33(3), 383-401.

Väänänen, J. (1979). Abstract logic and set theory. I. Definability. In Logic colloquium '78 (Mons, 1978), volume 97 of Stud. Logic Foundations Math. (pp. 391-421). Amsterdam: North-Holland.

Väänänen, J. (1985). Set-theoretic definability of logics. In Model-theoretic logics. Perspectives in mathematical logic (pp. 599-643). New York: Springer.

Vaught, R. (1973). Descriptive set theory in $L_{\omega_{1} \omega}$. In Cambridge Summer School in Mathematical Logic (Cambridge, England, 1971). Lecture notes in mathematics (Vol. 337, pp. 574-598). Berlin: Springer. 